tumor recurrence (Ca $125 \mathrm{p}=0.993$; HE4 $\mathrm{p}=0.311$; CEA $\mathrm{p}=0.417$ ). On ROC analysis only elevated Ca 125 serum levels were found to significantly indicate tumor recurrence $(p=0.031$; sensitivity $=80 \%$; specificity $=94.1 \%)$, while serum levels of CEA $(p=0.196)$ and HE4 $(p=0.754)$ were not significant predictors. Nevertheless, serum levels of investigated tumors markers were not correlated with time of tumor recurrence $(\mathrm{Ca} 125 \mathrm{p}=0.954$; HE4 $\mathrm{p}=0.952$; CEA $\mathrm{p}=0.702)$.

Conclusion* Elevated serum levels of $\mathrm{Ca} 125$ in the follow-up period of patients operated due to borderline ovarian tumors could be used as marker of tumor recurrence.

\section{DIFFERENT SURGICAL APPROACHES FOR EARLY-STAGE OVARIAN CANCER STAGING: A LARGE MONOCENTRIC EXPERIENCE}

${ }^{1} \mathrm{~S}$ Cianci, ${ }^{2} V$ Capozzi, ${ }^{3} \mathrm{~A}$ Rosati*, ${ }^{3} \mathrm{~V}$ Rumolo, ${ }^{3} \mathrm{G}$ Corrado, ${ }^{4} \mathrm{~S}$ Uccella, ${ }^{3} \mathrm{~S}$ Gueli Alletti, ${ }^{3} \mathrm{~A}$ Fagotti, ${ }^{3} \mathrm{G}$ Scambia. ${ }^{1}$ University of Messina, Department of Human Pathology of Adult and Childhood "G. Barresi", Italy; ${ }^{2}$ Ubniversity of Parma, Italy; ${ }^{3}$ Policlinico A Gemelli Foundation IRCCS, Italy; ${ }^{4}$ University of Verona, Italy

\subsection{6/ijgc-2021-ESGO.478}

Introduction/Background* Ovarian cancer is the third most frequent gynecological cancer. In early stage ovarian cancer (ESOC) comprehensive surgical staging is recommended.

Surgical staging is traditionally approached by laparotomy, although minimally invasive surgery can be a valid alternative in selected patients. This study aims to analyze the surgical and oncological outcomes of these three different approaches in a large series of patients.

Methodology We retrospectively included all histologically proven ESOC cases treated between January 2014 and December 2017. ESOC was defined as stage IA to IIB according to the 2018 FIGO staging system. Subjects were divided into groups 1,2 , and 3 , based on the surgical approach (open abdominal, laparoscopic, or robotic, respectively).

Result(s)* Within patients enrolled during the study period, 455 met the inclusion criteria.

No difference in intraoperative complications was recorded in the three groups

$(p=0.709)$. Conversely, a significant difference occurred in postoperative complications

( $16.2 \%$ vs. $3.8 \%$ vs. $11.1 \%$, in groups 1,2 , and 3 respectively, $\mathrm{p}=0.004)$. No difference was found in overall survival (OS) (32 vs. 31 vs. 25 months, $\mathrm{p}=0.481$ ) and disease-free survival (DFS) (26 vs. 29 vs. 24 months, $\mathrm{p}=0.178$ ) in groups 1 , 2 , and 3 , respectively.

\begin{tabular}{|c|c|c|c|c|c|}
\hline General Population & TOTAL & Open Abdominal & Laparoscopy & Robot & p value \\
\hline G1-G2* & 41 & 28 & 8 & 5 & $\mathrm{p}=0.008$ \\
\hline G3-G4** & 4 & 4 & 0 & 0 & $\mathrm{p}=0.112$ \\
\hline \multicolumn{6}{|l|}{ Fertility sparing } \\
\hline G1-G2 & 6 & 2 & 2 & 2 & $\mathrm{p}=0.318$ \\
\hline G3-G4** & 1 & 2 & 0 & 0 & $p=0.139$ \\
\hline \multicolumn{6}{|l|}{ Radical surgical staging } \\
\hline G1-G2 & 35 & 26 & 6 & 3 & $\mathrm{p}=0.076$ \\
\hline G3-G4" & 3 & 2 & 0 & 0 & $\mathrm{p}=0.156$ \\
\hline
\end{tabular}

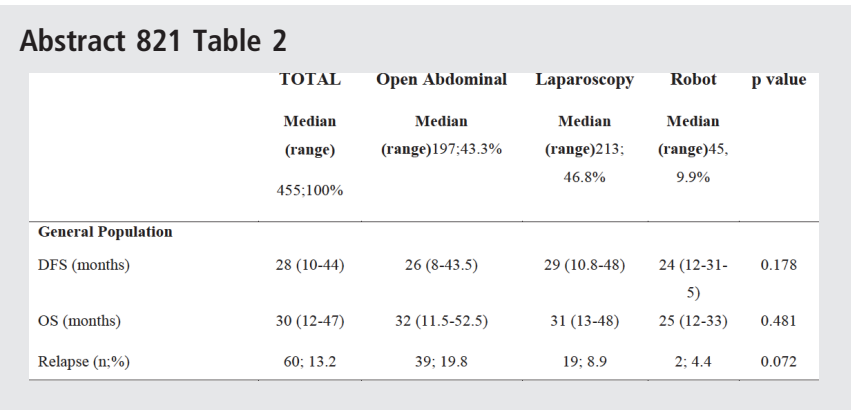

At univariate analysis FIGO stage I $(p=0.004)$ showed a lower recurrence rate compared to FIGO stage II.

Conclusion* No significant difference was found in OS and DFS among the three groups (open, laparoscopic, and robotic). The minimally invasive approach showed lower rate of complications than the laparotomic one, thus it should be preferred in selected patients.

\section{COMPARATIVE STUDY OF LAPARASCOPY SCORING AND LAPARATOMY STAGING IN ADVANCE OVARIAN CANCER}

F Behnamfar*, S Rouholamin, M Nazemi, FZS Allameh, F Sabet, L Mousavi Seresht. Isfahan University of Medical Sciences

\subsection{6/ijgc-2021-ESGO.479}

Introduction/Background* comparative study between laparascopic scoring and laparatomy scoring in patient with advanced ovarian cancer

Methodology This prospective study conducted between march2020 and march2021. Participant in the study were 27 patients with advanced ovarian cancer who underwent laparoscopy and laparotomy scoring at hospitals affiliated to Isfahan University of Medical Science. Predictive index value (PIV) score (range: 0-14) was calculated for all patients. Patients with PIV scores $<8$ were offered primary cytoreductive surgery and those with score $\geq 8$ received NACT)neoadjuvant chemotherapy). Patients who underwent primary cytoreductive surgery received a second PIV score at the time of their laparotomy and concordance between two PIV scores were calculated. All patients had primary surgery at the same day as laparoscopy. Residual disease following primary cytoreductive surgery was documented for each patient. PPV was used to determine the ability of the PIV score at laparoscopy to predict R0 at primary cytoreductive surgery.

Result(s)* 27 patients underwent laparoscopic scoring, 25 patient $(92 / 5 \%)$ had a PIV score $<8$ and 2 (7/5\%) had a PIV score $\geq 8$. There was overall $92 \%$ concordance between PIV scores at laparoscopy and laparotomy. Concordance scores by location were: bowel infiltration $76 \%$, mesenteric disease 92\%, liver surface involvement 96\%, omental disease92\%, diaphragm disease 96\%, stomach infiltration 100\%, peritoneal carcinomatosis 96\%. A laparoscopic PIV score of $<8$ had a PPV of $92 \%$ at predicting R0 at primary cytoreductive surgery.

Conclusion* Laparoscopic scoring allowed for a more personalized approach to the management of patients with advancedstage ovarian cancer at our institution. It resulted in an objective triage of patients to primary cytoreduction or NACT, and 